\title{
Game theory in models of pedestrian room evacuation
}

\author{
S. Bouzat and M. N. Kuperman \\ Consejo Nacional de Investigaciones Científicas y Técnicas \\ FiEstIn, Centro Atómico Bariloche (CNEA), (8400) Bariloche, Río Negro, Argentina.
}

\begin{abstract}
We analyze the pedestrian evacuation of a rectangular room with a single door considering a Lattice Gas scheme with the addition of behavioral aspects of the pedestrians. The movement of the individuals is based on random and rational choices and is affected by conflicts between two or more agents that want to advance to the same position. Such conflicts are solved according to certain rules closely related to the concept of strategies in Game Theory, cooperation and defection. We consider game rules analogous to those from the Prisoner's Dilemma and Stag Hunt games, with payoffs associated to the probabilities of the individuals to advance to the selected site. We find that, even when defecting is the rational choice for any agent, under certain conditions, cooperators can take advantage from mutual cooperation and leave the room more rapidly than defectors.
\end{abstract}

\section{INTRODUCTION}

The problem of enclosed pedestrian evacuation has been studied from various points of view. On the one hand it presents academic interest because of its similarity with makeshift linked to granular media [1]. On the other hand, understanding the dynamics of the movement of pedestrians and anticipating the problems that may arise in an emergency situation is critical in the design of large spaces that will be occupied by many people [2]. The efficient evacuation of the occupants of such places under a state of emergency is fundamental when trying to minimize the negative effects of panic and confusion, clogging and avalanches. Predicting evacuation patterns is one of the first steps towards this goal. Evacuation is only a particular aspect of a broader problem: the pedestrian movement.

Pedestrian dynamics has been extensively studied from a theoretical [3-7] and experimental point of view $8-12$.

The associated pedestrian flow is usually modeled as a many-body system of interacting individuals. The literature on this subject is rather extensive [13 22], exposing several different approaches to the problem. In [11], the authors introduce the active walker model to describe human trail formation and they show that the pedestrian flow system exhibits various collective phenomena interpreted as self-organized effects. In [23] the author suggest that the behaviors of pedestrian crowds are similar to gases or fluids. Other authors prefer the formalism of cellular automata to frame their models [24]. This is the approach we are going to adopt in the present work.

In the present Lattice Gas [25] model, $N$ pedestrians are set on the sites of a $L \times W$ lattice, with the restriction that each site cannot hold more than one walker. The pedestrians move to empty sites according to a preferential direction dictated by the need of escaping from the room [8, 26]. Conflicts between agents that want to get to the same position are solved using specific game rules and taking into account previously defined strategies (of cooperation or defection) which represent the characters of the agents. Hence, the considered pedestrian dynamics include games between agents which affect their possibilities of leaving the room.

Real pedestrians are entities much more complex than automata with the sole idea of escaping from a room. One of the most difficult aspects of modeling pedestrian flow is to simulate the effects of subjective aspects that affect the interaction among the pedestrians [12, 27 -30]. Most of the models have focused on modeling the flow of individuals in pure mechanistic approaches but the behavioral reaction of the evacuees during their movement has not been so far investigated in detail. By including a game dynamics in the resolution of conflicts between pedestrians, the present work aims at opening a door to the analysis of this important and rather complex feature.

\section{X 2 SYMMETRIC GAMES}

First, we want to introduce some basic concepts of game theory and $2 \times 2$ symmetric games that are relevant for the pedestrian dynamics that we will consider. A two players game can be characterized by the set of the strategies that the players can adopt and by the payoff received by each strategy when confronting any other. If the game is symmetric, i.e. both players have access to the same set of strategies and payoffs, the information can be uploaded in an $n \times n$ matrix, with $n$ the number of strategies. In 1966, M. Guyer and A. Rapoport [31] cataloged all the $2 \times 2$ games. There are twelve symmetric games, eight of them are trivial, in the sense that there is no conflict of interest as both players prefer the same outcome. The remaining four games represent four distinct social dilemmas.

In a $2 \times 2$ game there are only two different strategies that can be defined as cooperative (C) and defective (D). 
We can consider only relative payoff values, and this let us with four generic payoffs represented in the following matrix

\begin{tabular}{|c|c|c|}
\hline & $\mathrm{C}$ & $\mathrm{D}$ \\
\hline $\mathrm{C}$ & $C C$ & $C D$ \\
\hline $\mathrm{D}$ & $D C$ & $D D$ \\
\hline
\end{tabular}

Here, each matrix element indicates the payoff of a player adopting the strategy at the file when competing with a player adopting the strategy at the column. $C C$ is the payoff to each of the two players, when both cooperate; $D D$ is the payoff when both defect. When one player cooperates and the other defects, the payoffs of the cooperator and the defector are $C D$ and $D C$ respectively.

The relative values of the four payoffs characterize the whole family of symmetric $2 \times 2$ games.

In order to be consistent to the names assigned to the strategies, the payoff $C C$ must be preferred to $C D$, meaning that a cooperation from the opponent is always preferable to defection. Also, $D C$ must be better than $D D$. Still, there is something missing to define a dilemma. If defection is bad, it is natural to avoid it, unless there is a temptation to defect. There are three situations in which this can occur. Either there is an incentive to defect when the other player cooperates $(D C>C C)$, or there is an incentive to defect when the other player defects $(D D>C D)$, or both.

There are four different games that fulfill at least one of the aforementioned conditions.

- Prisoner's Dilemma: $D C>C C>D D>C D$

- Stag Hunt: $C C>D C>D D>C D$

- Deadlock: $D C>D D>C C>C D$

- Chicken: $D C>C C>C D>D D$

We want to focus on the two first cases. In particular, the Prisoner's Dilemma (PD) represents situations in which obtaining cooperation is difficult because of the increased individual incentives to defect. Despite that players can realize that they would be better if they both cooperate than if they both defect, defecting is individually the best choice. It is a dominant strategy. Under this scenario, organizational cooperation involves organizing the individuals to work towards a common goal even if they have to give up personal incentives to defect.

In turn, Stag-Hunt (SH) represents a situation in which coordination is difficult because of the uncertainty about what the opponent will do. Unlike in $\mathrm{PD}$, both players realize that they are best off when they coordinate on $(\mathrm{C}$, $\mathrm{C})$, but do not want to select $\mathrm{C}$ if they think that the other player will not do so as well. Therefore, organizational coordination involves be convinced that others will work towards the common goal as well, in which case it is individually rational for everyone to do so.

\section{MODEL}

We analyze the evacuation of a rectangular room of size $L \times W$ with a single door of length $L_{d}$ located at the center of one of the walls. We consider a discrete time and space dynamics in which the pedestrians (or agents) can occupy the sites of a square lattice an can perform jumps between first neighbor sites according to certain rules. When more than one agent attempts to jump to the same site at the same time a conflict occurs. To solve the conflict the involved agents (which can be between 2 and 4) play a game using previously defined strategies. As a results of the game, either there can be a winner which finally gets to the desired position, or there can be no winner and all the involved agents loose their opportunity to move at that time step.

The discrete sites are labeled as $(x, y)$ with $1 \leq x \leq L$ and $1 \leq y \leq W$. The sites with $x=1, x=L$ and $y=W$ belong to the walls and cannot be occupied by agents. The sames occurs for the sites with $y=1$ excepting for those with $x_{l} \leq x \leq x_{r}$ that correspond to the door exit. We consider a symmetric position of the door setting $x_{l}=L / 2-L_{d} / 2$ and $x_{r}=L / 2+L_{d} / 2$. For the sake of simplicity, throughout the paper we consider $L_{d}=L / 10$.

We consider an initial density of agents $0 \leq \rho \leq 1$, corresponding to an initial number of agents equal to Int $(\rho \times$ $\mathrm{L} \times \mathrm{W})$. The agents are placed at random positions $1<x<L, 1<y<W$ at $t=0$. Then, at each time steps, the dynamics involves three stages. First, every agent chooses a neighbor site where to attempt to jump. Second, all the conflicts are identified and solved according to the game rules. Finally, the winners of the conflicts (as well as the agents that can move without conflict) jump to their desired positions. The agents that reach the door are taken out of the system. The simulation ends when all the agents have abandoned the room. In the next paragraphs we explain each of the three instances in detail . 
First stage: Choosing the site where to attempt to jump

Each agent can attempt to jump to any of the four neighbor sites which we simply label as up, down, left and right (see figure 1.a). With probability $R(0 \leq R \leq 1)$, the direction of the jump attempt is chosen at random. Conversely, with probability $1-R$ the direction of jump attempt is chosen according to a desired (or rational) direction which is defined for each agent pointing essentially toward the door in a way that we explain in detail below. Importantly, the angle $\alpha$ defining such desired direction is continuous between 0 and $2 \pi$. Then the desired direction is projected on the discrete allowed directions in order to define the probabilities for attempt of jumps. This is done as follows. For instance, consider the case depicted in figure 1.a. In such a case, we define the probabilities for attempt for a jump to the up, down, right and left directions as $p_{u}=R / 4, p_{d}=R / 4+(1-R) \cos (\alpha) / Z, p_{r}=R / 4$ and $p_{l}=\frac{1}{4} R+(1-R) \sin (\alpha) / Z$, respectively. Here, $Z=|\cos (\alpha)|+|\sin (\alpha)|$ is a normalization constant required to have $p_{u}+p_{d}+p_{r}+p_{l}=1$. Note that, in this case, $p_{u}$ and $p_{r}$ contain only the term proportional to $R$ describing the random way of choosing, while $p_{d}$ and $p_{l}$ include also a term which is proportional to $(1-R)$ and to the projections of the desired direction. Now, considering an arbitrary desired direction defined by an angle $0 \leq \alpha \leq 2 \pi$ increasing clockwise from $\alpha=0$ (corresponding to the down direction), we have the general definition

$$
\begin{aligned}
p_{u} & =\frac{1}{4} R+(1-R) \frac{(-\cos (\alpha))(1-\operatorname{sgn}(\cos (\alpha))}{2 Z} \\
p_{d} & =\frac{1}{4} R+(1-R) \frac{\cos (\alpha)(1+\operatorname{sgn}(\cos (\alpha))}{2 Z} \\
p_{r} & =\frac{1}{4} R+(1-R) \frac{(-\sin (\alpha))(1-\operatorname{sgn}(\sin (\alpha))}{2 Z} \\
p_{l} & =\frac{1}{4} R+(1-R) \frac{\sin (\alpha)(1+\operatorname{sgn}(\sin (\alpha))}{2 Z} .
\end{aligned}
$$

Note that the desired or rational direction contributes only to the probabilities of jumping to the discrete directions on which it has a positive projection. Moreover, the probability $R$ measures the randomness of the motion in such a way that for $R=0$ only the two discrete direction defined by the desired direction are allowed, while for $R=1$ all four directions have equal probabilities.
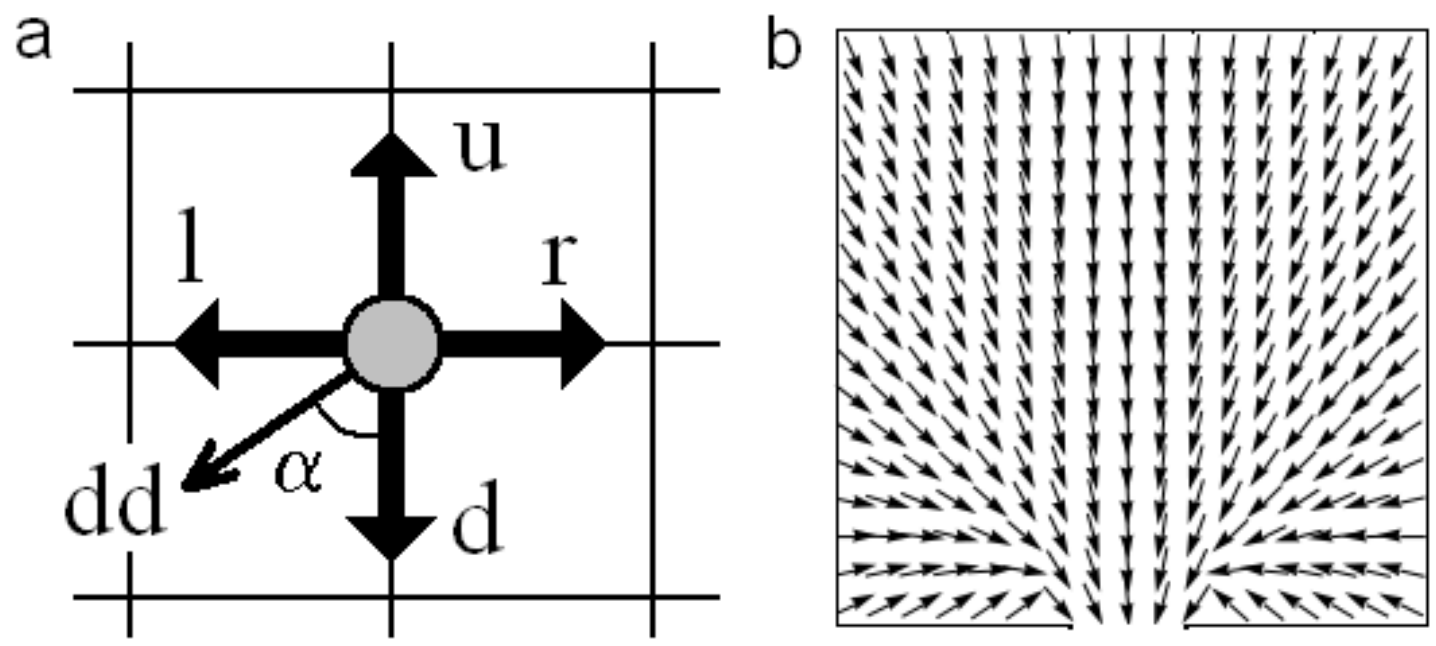

FIG. 1: Model for agents motion. a) Allowed directions (up, down, left, right) for the motion of an agent (central circle), and desired direction (dd). b) Field of desired direction in a square room.

The desired direction is defined through a target position $\left(x_{T}, y_{T}\right)$ toward which the agents point. For an agent located at $x, y$ we set $x_{T}=(L+1) / 2$ (independently of $x$ and $\left.y\right), y_{T}=-L / 10+2 L / 5\left(-y / \sqrt{(y-L / 2+5)^{2}+y^{2}}+\right.$ $\left.\sin \left(\tan ^{-1}(a)\right)\right)$ for $y<a\left(x-x_{r}\right)$ or $y<-a\left(x-x_{l}\right)$ and $y_{T}=-L / 10$ otherwise. Here, $x_{r}$ and $x_{l}$ are the right and left limits of the door and $a=3$. Figure 1.b shows the field of desired directions for a square room. The dependence of $y_{T}$ on $(x, y)$ is chosen in order to produce a recirculation pattern that prevents the agents to remain "trapped" close to the bottom wall. This can be considered as equivalent to an effective repulsion exerted by the bottom wall. Such recirculation pattern is important to get a realistic escape dynamics as the one shown in figure 2 . The consideration 
of a fixed target position (independent of the agent position) produces non realistic dynamics close to the $y=1$ wall. For instance, for a fixed target position at the center of the door, an agent located at the exit would walk along the door until it reaches its center instead of getting out of the room immediately.

Now we can finally explain the detailed procedure for determining the attempt of jump for each agent at each time step. First, the desired direction and the probabilities given in equation (1) are computed. Second, using the probabilities (1), the agent performs two attempts of finding an empty neighbor site for the jump. This means that one of the four allowed directions is selected according to the probabilities (1). Then, in case the corresponding neighbor site is empty, it is marked as chosen for an attempt of jump by the considered agent. Meanwhile, if the neighbor site is not empty, the procedure of selecting one of the four allowed directions according to the probabilities (11) is repeated once. In case that the same or another non empty neighbor site is selected, the agent will not move at that time step.

\section{Second stage: solving the conflicts}

When a given site is chosen by more than one agent on their attempt to jump, we say that there is a conflict at that site. Thus, once all the pedestrian have chosen their sites where to attempt to jump (excepting those that have lost their opportunity of jumping due to having chosen a non empty site), all the conflicts have to be identified and solved in order to determine the agents that will be able to move at the time step. The conflicts and their solutions imply thus an effective interaction between the agents which affects the dynamics.

A conflict can involve two, three or four agents, due to the existence of four allowed directions for arriving to a given site. Each conflict will be solved through a game. As a result of the game, a winner may be selected to jump to the desired site, while the rest of the players (the losers) will lose their opportunity to move at that time step. It is important to stress that, with certain probability that we later indicate, a game can have no winner. In such a case, all the players will lose their opportunity to move.

The definition of the game demands the consideration of strategies for the players. We consider that from the beginning of the dynamics each pedestrian adopts an attitude that can be either cooperative (C) or a defective (D). At the moment of the conflict, and according to the strategies chosen by each of the individuals involved in the conflict, the players will be assigned a probability to win, i. e. to jump to the desired site. In case of a competition between two individuals the considered probabilities are loaded into the following matrix, that is analogous to a payoff matrix.

$$
\begin{array}{|c|c|c|}
\hline & \mathrm{C} & \mathrm{D} \\
\hline \mathrm{C} & \frac{1}{2} & 0 \\
\hline \mathrm{D} & \frac{1}{P} & \frac{1}{2 P} \\
\hline
\end{array}
$$

Here $P \geq 1$ is a parameter that measures the conflictive attitude of the defectors, which makes them focus more on the competition than on the possibilities of movement. Thus, the matrix summarizes our assumptions for the two players games, which are the following. First, in a two cooperators game there is always a winner (that jumps to the desired position) which is chosen at random between the two players. Second, in a game between a cooperator and a defector, the cooperator has no chance to advance while the defector advance with probability $1 / P$. Third, in a game between two defectors, each of the agents has probability $1 /(2 P)$ of becoming the winner. Note that the larger the value of $P$, the lower the probabilities of stepping of the defectors, both when interacting with defectors and with cooperators. In particular, in a game with at least one defector, the existence of a winner is ensured only for $P=1$. Thus, values $P>1$ are used to model situations in which defectors sometimes loose their opportunity to move due to their conflictive attitude. In this sense, $P$ is a punishment to defection. Defection generates aversion among cooperators, who when confronting a defector cooperate but with some resilience. The interaction between defectors is competitive and non cooperative leading to a delay in the solution of any conflict that may arise.

The two players game considered is straightforwardly analyzable in terms of game theory. We observe that when $1 \leq P<2$ the matrix is analogous to the payoff matrix of the Prisoner's Dilemma, while for values of $P$ higher than 2 , the analogous situation is the Stag Hunt. As mentioned before, in both cases there is an incentive to defect. While in the first case D is clearly a dominant strategy, this is not true for the Stag Hunt. Still, in both cases, cooperators can only take advantages from mutual cooperation, that is more attractive when $P>2$. The lower bound for $P$ is imposed to fulfill the requirement $2 C C>D C+C D$ that prevents alternating cooperation and defection in an iterative game.

The example can be generalized for encounters of $n$ players by defining the probabilities of winning of a $\mathrm{C}$ and a $\mathrm{D}$ player in the cases in which all the rest are cooperators, and in the cases in which the player $(\mathrm{C}$ or $\mathrm{D})$ compete against $m<n-1$ cooperators and $n-1-m$ defectors. We consider such probabilities defined by following matrix: 


\begin{tabular}{|c|c|c|}
\hline & $(\mathrm{n}-1) \mathrm{C}, 0 \mathrm{D}$ & $\mathrm{m} \mathrm{C},(\mathrm{n}-1-\mathrm{m}) \mathrm{D}$ \\
\hline $\mathrm{C}$ & $\frac{1}{n}$ & 0 \\
\hline $\mathrm{D}$ & $\frac{1}{P}$ & $\frac{1}{(n-m)^{2} P}$ \\
\hline
\end{tabular}

The contents of the matrix can be summarized as follows. In a game between $n$ cooperators and no defectors, there is always a winner. One of the players is randomly chosen to move (with probability equal to $1 / n$ ). In a game with at least one defector, the existence of a winner is not ensured. In this case, the cooperators always loose and and each defector has probability $\frac{1}{(n-m)^{2} P}$ of winning, with $m$ the number of cooperators in the game. The probability of having a winner is $\frac{1}{(n-m) P}$.

\section{Third stage: movement of agents}

As stated before, once all the conflicts have been solved, all the winners as well as all the agents that can move without conflict are finally moved to their selected sites. In case that one agent gets to a position with $y=1$ (i.e. it reaches the exit), it is taken out of the system and the number of escaped agents is increased by one.

\section{RESULTS}

We characterize the evacuation dynamics of the system by computing the mean exit time which is defined as the average over realizations of the number of time steps required to evacuate completely the room. We also study the time dependence of the mean number of escaped agents, i.e. the average over realizations of the number of agents that have reach the exit at a given time.

Before considering the general case of a population with both cooperators and defectors, we study the extreme cases in which all the agents have the same strategy.

\section{Only Cooperators: the random game case}

First we consider a population in which all the agents are cooperators. In this case, any conflict leads to a random game in which there is always a winner. Namely, for a game between $n$ cooperators, each of them has a probability $1 / n$ of becoming the winner and, thus, moving to the desired site. Note that the parameter $P$ results irrelevant. In Figure 2 we show the positions of all the agents at six instances of the evolution for a single realization of the dynamics for a system of $L=W=50$.

Figure (3) shows results for the mean exit times and the number of escaped agents as a function of time for the evacuation dynamics from a square room considering different system parameters $\rho, R$ and $L$. In Fig.(3).a we see that the mean exit time increases with $R$ more rapidly than exponentially. This means that the randomness of the dynamics strongly slows the evacuation process. Note that the randomness may be associated to an uncertainty of the agents in their knowledge of the position of the door. We can also see that the mean exit time increases with the initial density, as could be expected. Figure (3).b shows that such a growth with $\rho$ is linear at fixed $R$.

In Fig.(3).c we study the dependence of the mean exit time on the system size. We see that the growth is slower than exponential and faster than linear.

Finally, Fig.(3).d shows the evolution of the mean number of escaped agents for different values of $\rho$ and $R$. Here, we see that the growth is linear along most of the evolution. Small deviations from the linear regime occur for very short times and very long times. This is due to the fact that the flux of pedestrian out of the room is controlled mainly by the local density at the exit (and by the parameter $R$ which is constant). The local density at the exit at small times coincides with $\rho$, then it increases until it reaches a quasi-stationary value. Such quasi-stationary density determines the slope of the linear growth of the evacuation profile. Finally, at large times, when the evacuation process is about to finish, the density at the exit decreases and the flux at the door is reduced. Fig.(3).d also shows that the slope of the linear growth is essentially independent of the initial density. This indicate that the quasi-stationary value of the density at the door depends only on $R$, as could be expected. 

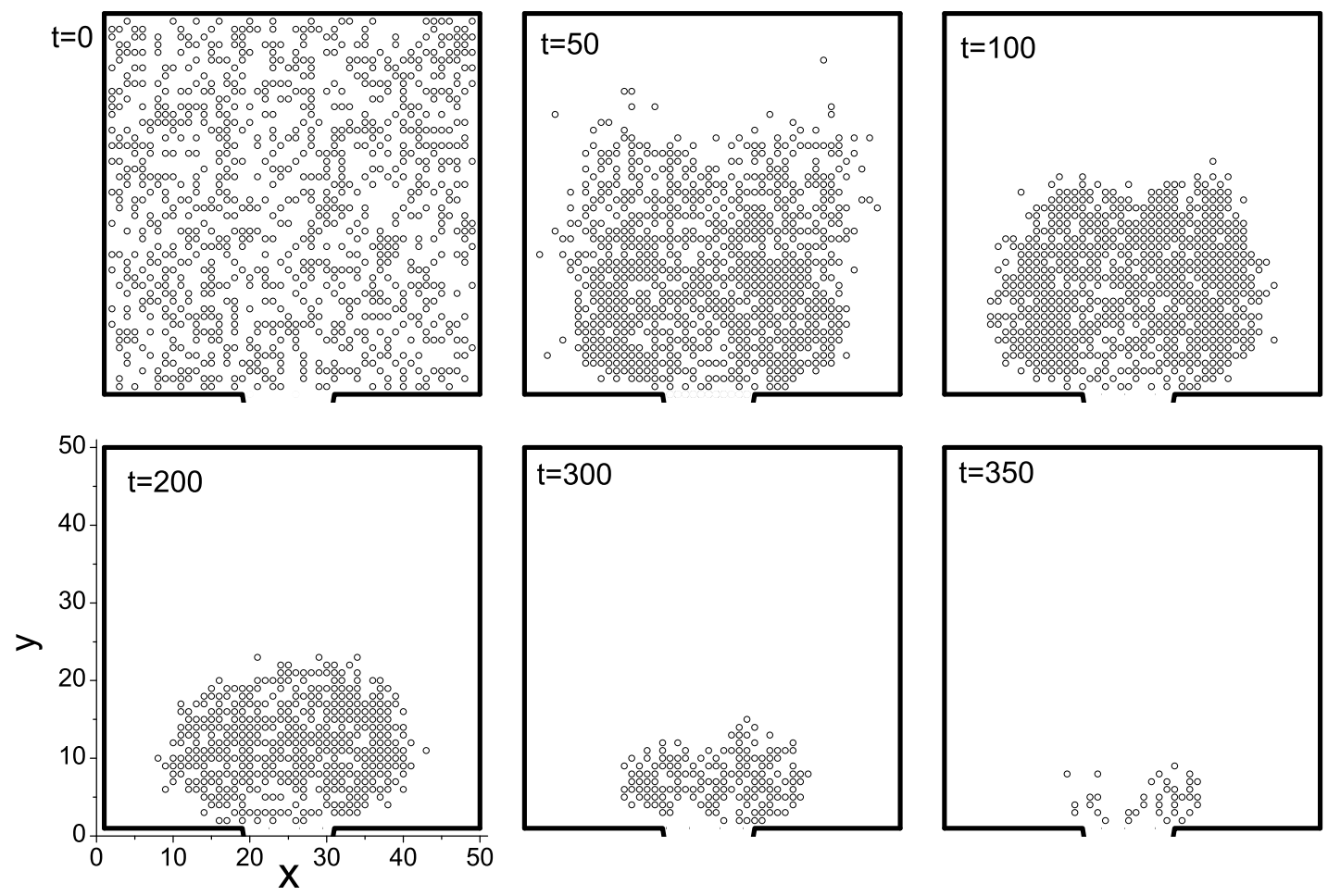

FIG. 2: Room evacuation for a population with only cooperators. Parameters $L=W=50, \rho=0.4, R=0.3$.

\section{Only Defectors}

Now we consider a population in which all the agents are defectors. This is an extreme case opposite to the one considered in the previous subsection. We focus on the dependence of the results on the parameter $P$ which now becomes relevant as it rules the probabilities of motion resulting from all the conflicts.

Figure (4).a shows the mean exit time as a function of the initial density $\rho$ for different values of $P$, while Figure (4).b shows the mean exit time as a function of $P$ for different values of $\rho$. For the sake of comparison, in both insets we also include the results for the only-cooperators case studied in the previous subsection. It can be seen that the mean exit time grows linearly both with $\rho$ (Fig.(4).a) and with $P$ (Fig.(44).b). Moreover the mean exit time for the only-collaborators case is always smaller than that for the only-defector case at any value of $P$. This last fact can be understood as a consequence of the delays for stepping after the conflicts, which are present only for games with defectors.

\section{Heterogeneous Populations}

Now we study the evacuation problem for heterogeneous populations with both collaborators and defectors.

In Figure 5.a we show the mean exit time as a function of the initial fraction of defectors $\rho_{D}$ for fixed values of $\rho, R$ and $L$ considering different values of $P$. It can be seen that for $P=1$ the exit time is almost independently of $\rho_{D}$. This is so because in this case the penalization for defectors is null excepting for games with more than two defectors, which are likely only at large $\rho_{D}$. At larger values of $P$ the exit time grows approximately linearly with $\rho_{D}$. In Figure 5.b we show the normalized exit time, defined as $\left(t\left(\rho_{D}\right)-t\left(\rho_{D}=0\right)\right) / t\left(\rho_{D}=1\right)$, where $t\left(\rho_{D}\right)$ is the mean exit time obtained for an initial fraction of defectors $\rho_{D}$. The plot shows us that the curves for large enough $P$ are close to collapse, indicating thus the approximately linear behavior with $\rho_{D}$. However a close look to the figure reveals that the dependence on $\rho_{D}$ may be exactly linear only for a value of $P$ close to $P=2$ (i.e. the limit between $\mathrm{PD}$ and $\mathrm{SH}$ games), while it is superlinear for smaller values of $P$ (PD game) and slightly sublinear for larger values of $P$ (SH game).

One important questions we pose is whether cooperators can or cannot take advantages from mutual cooperation 

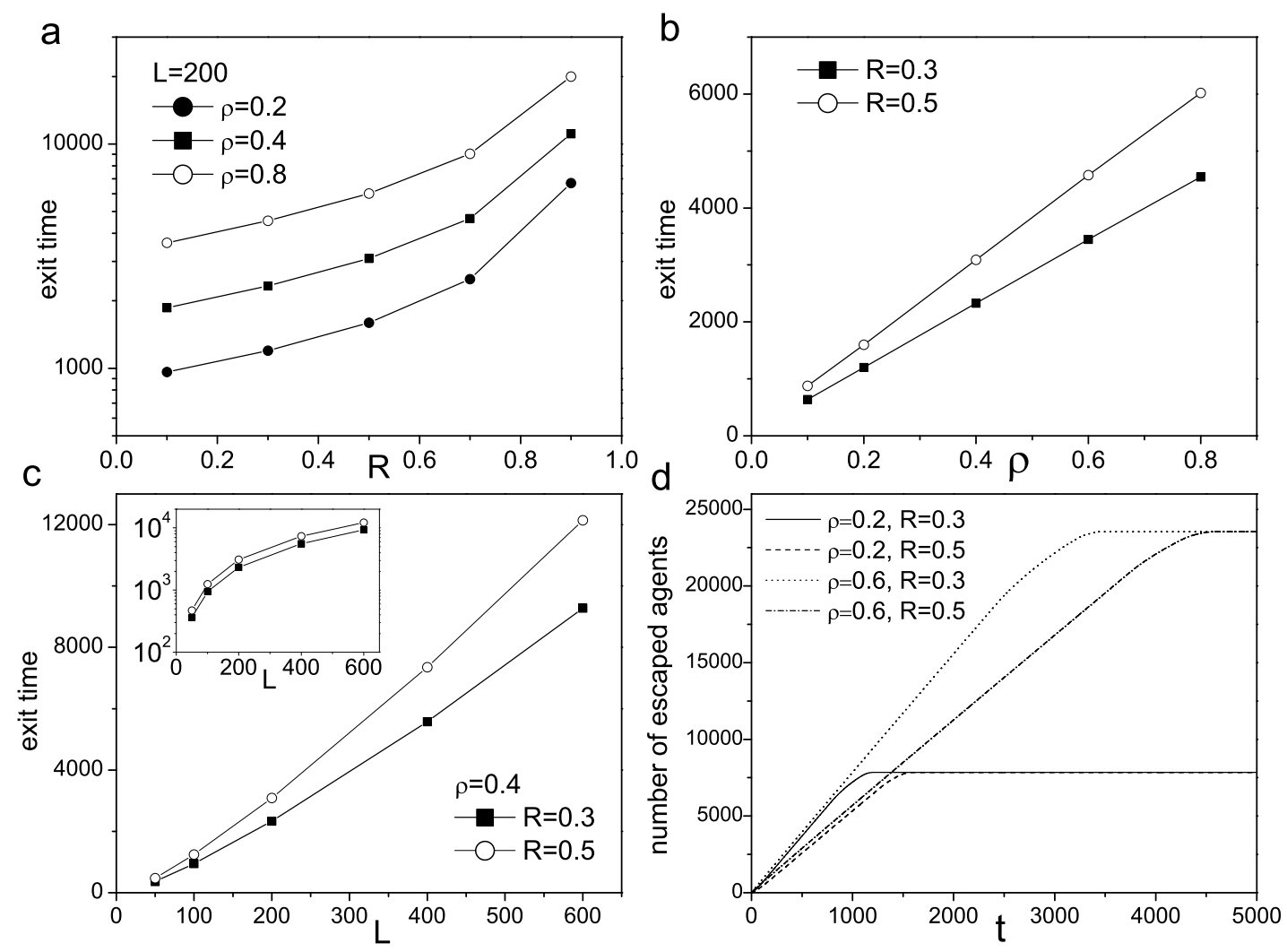

FIG. 3: Results for a population of only cooperators in a square room $(L=W)$. a) Exit time as a function of $R$ for different values of $\rho$ and $L=200$. b) Exit time as a function of $\rho$ for different values of $R$ and $L=200$. c) Exit time as a function of $L$ for different values of $\rho$. The small inset shows the same curves in logarithmic scale. d) Evolution of the number of escaped agents for different values of $\rho$ and $R$ for $L=200$.

as it was verified in other systems where cooperation arise as an emerging phenomena [32 41]. With this goal in mind we characterize the dynamics of the system by three quantities, all of them aiming at revealing the relative success of the cooperators in finding the exit. First, we sample the composition of the population inside the room, comparing the instantaneous fraction of cooperators with the initial one. We define a normalized instantaneous fraction of cooperators in the room as

$$
\rho_{c}^{i}(t)=\frac{\rho_{c}(t)-\rho_{c}(0)}{\rho_{c}(0)}
$$

where $\rho_{c}(t)$ is the fraction of cooperators in the room at time $t$. A departure of the derivative of $\rho_{c}^{i}(t)$ from zero indicates that the leaving individuals do not represent a random sample of the population in the room. A positive (negative) value of $\rho_{c}^{i}(t)$ indicates that the population in the room has a greater (lower) fraction of cooperators than the initial. Meanwhile, a positive (negative) derivative of $\rho_{c}^{i}(t)$ indicates that defectors (cooperators) are being more successful in finding the exit. Figure [ show the time behavior of this quantity for two values of the initial density of defectors and several values of $P$ for two different values of $\rho$. We observe that for small values of $P$ we have $\rho_{c}^{i}>0$ throughout the evolution meaning that the defectors clearly surpass the cooperators in finding the exit. Note that, not only $\rho_{c}^{i}(t)$ is positive but its derivative increases with time, indicating the continuous increment of the fraction of cooperators in the room. But as $P$ increases and even still in the PD regime $(P=1.8$ in the figure), the values of $\rho_{c}^{i}$ become negative meaning that the cooperators start to profit from mutual cooperation. While defectors loose time in futile arguments, the cooperators leave the room. $\rho_{c}^{i}(t)$ and its derivative are negative, reflecting the ability of cooperators to reach the exit. We have studied several initial configurations with different initial conditions including varying values for the initial density of individuals, the initial fraction of cooperators and defectors and several sizes. With some small variations, we have found qualitatively the same results indicating the prevalence of defectors at small $P$ and the prevalence of collaborators at large $P$.

To complement this measure, we analyze the strategy of the individuals exiting the room at each time step and 

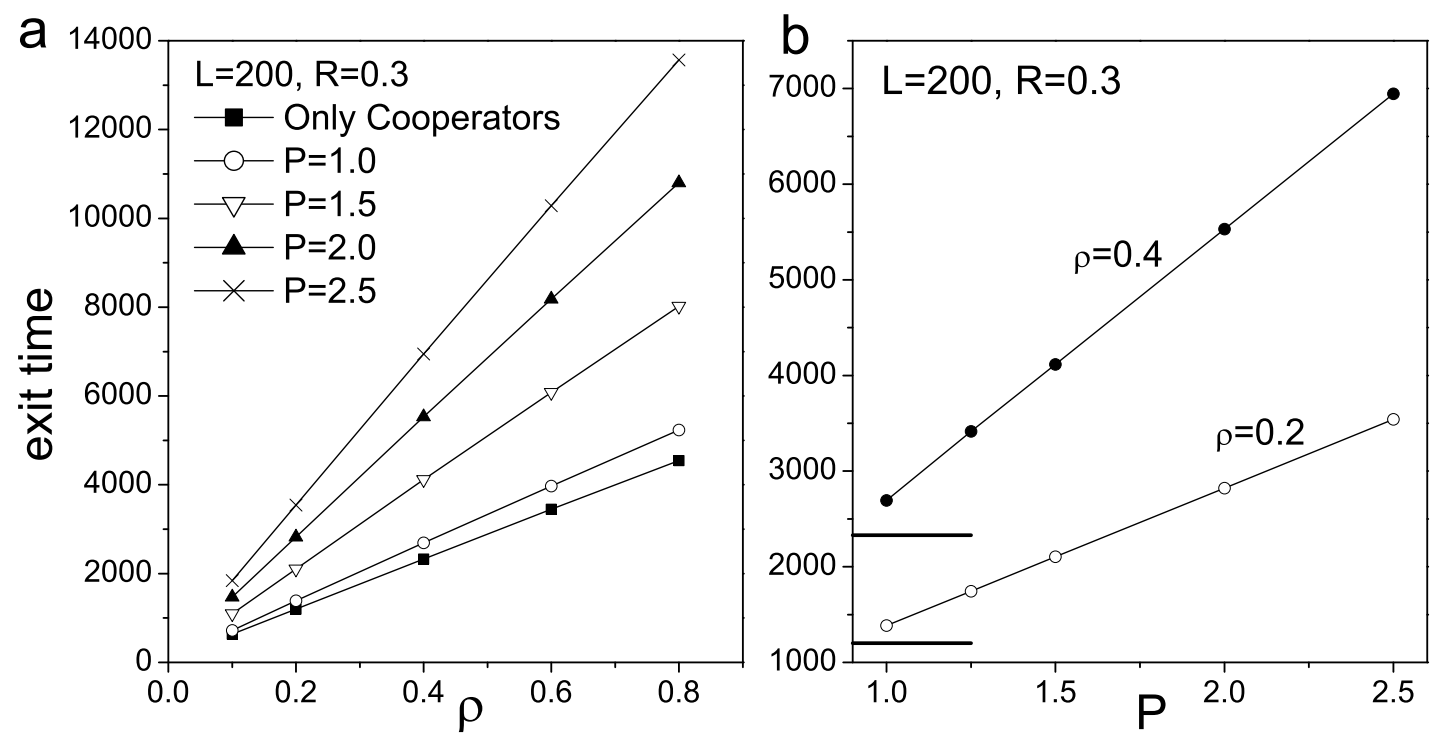

FIG. 4: Evacuation dynamics for systems with only defectors. a) Mean exit time as a function of the initial density of agents $\rho$ for different values of $P$. For the sake of comparison we also indicate the results for a system with only cooperators (squares). b) Mean exit time as a function of $P$ for different values of $\rho$. The segments on the left indicate the values for systems with only cooperators.
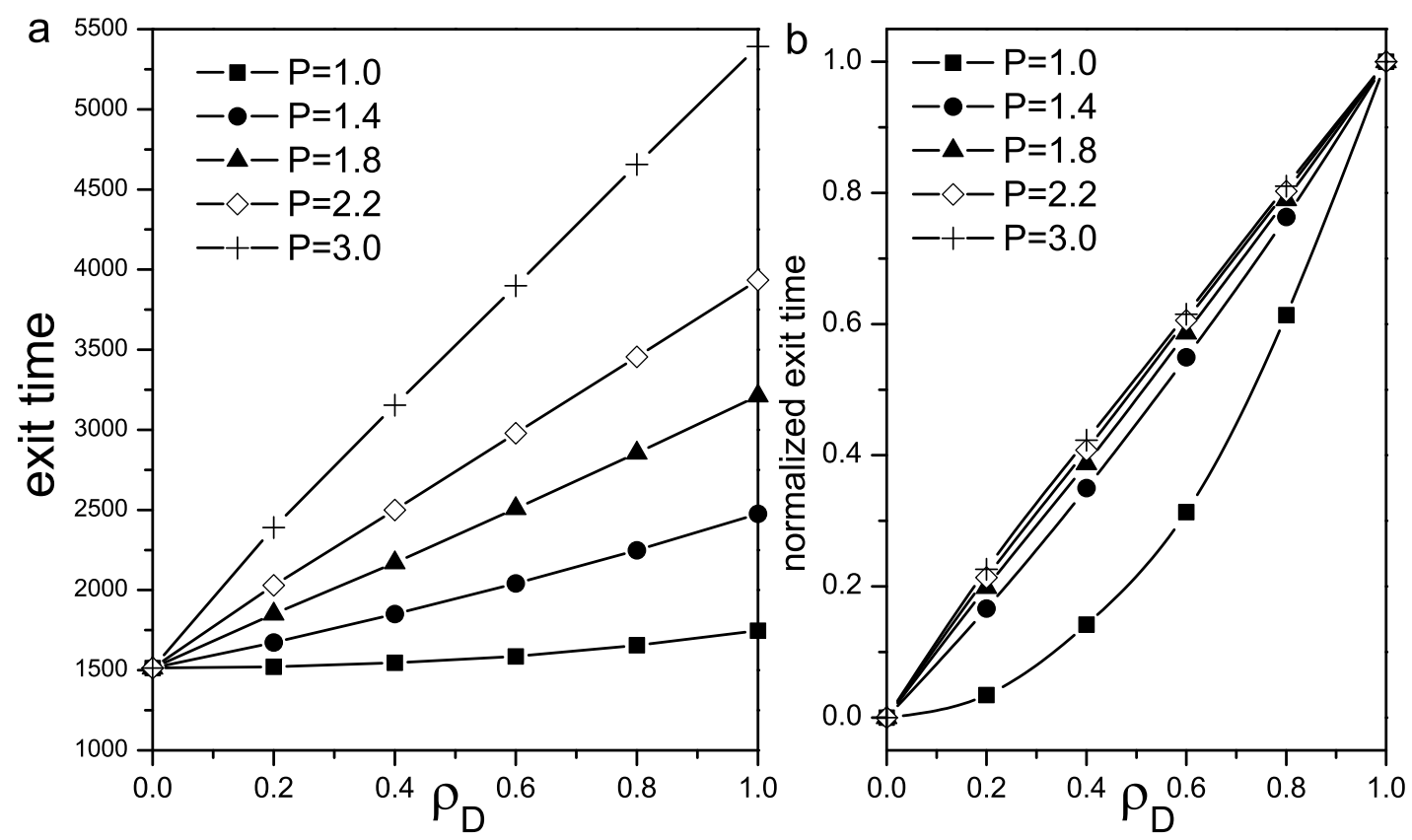

FIG. 5: Evacuation dynamics for heterogeneous populations. a) Exit time as a function of the initial fraction of defectors for different values of $P$. b) Normalized exit time as a function of the fraction of defectors for the same systems as in (a). All the calculations are for $L=200, \rho=0.4$ and $R=0.3$.

calculate the fraction of cooperators among them. We compare this fraction with the corresponding to the cooperators remaining in the room and define the normalized fraction of exiting cooperators as

$$
\rho_{c}^{e}(t)=\frac{\eta_{c}(t)-\rho_{c}(t)}{\rho_{c}(t)} .
$$

Here $\eta_{c}(t)$ is the ensemble averaged fraction of cooperators among exiting individuals at time $t$. Figures 7 show the 


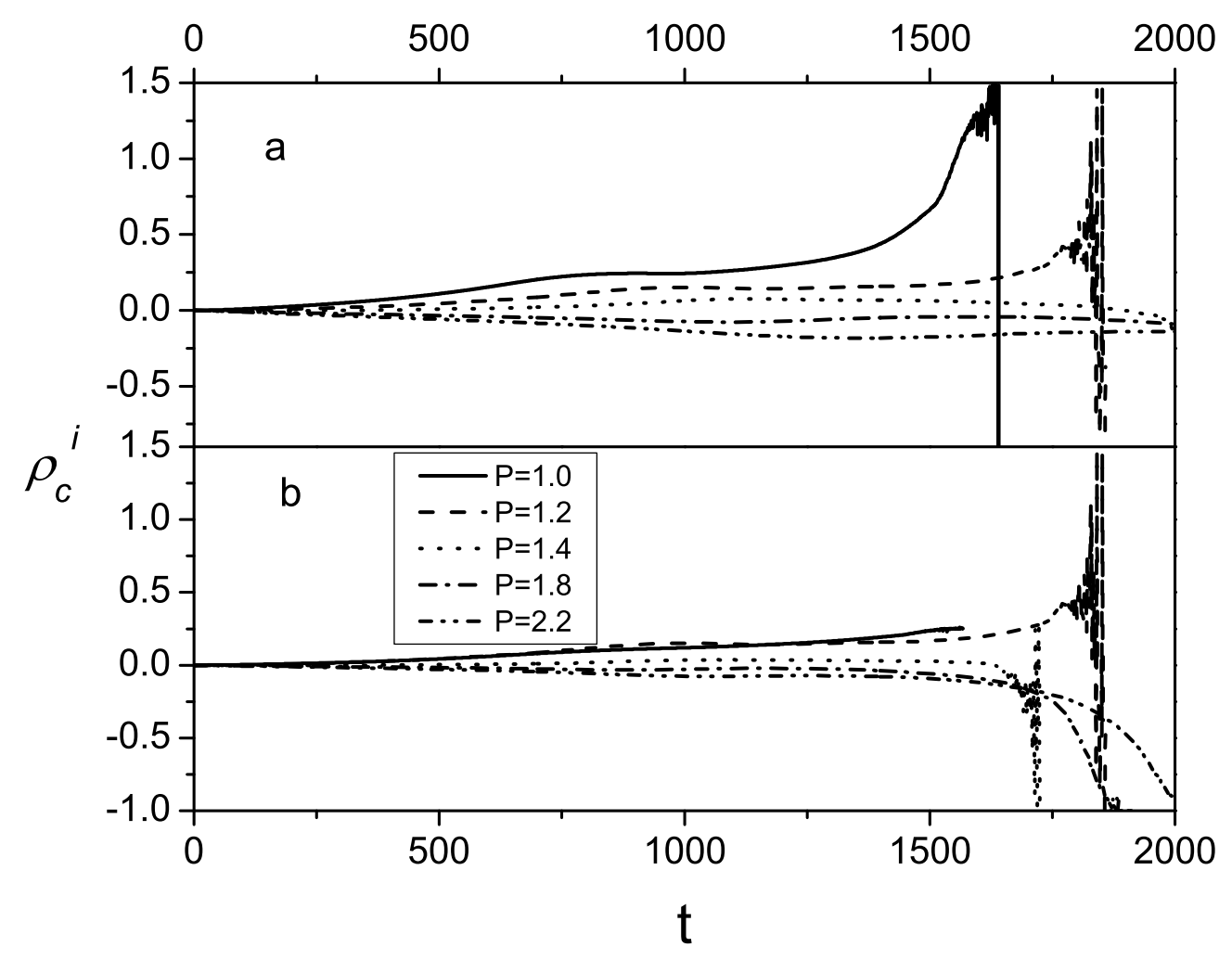

FIG. 6: Normalized instantaneous fraction of cooperators within the room for different values of $P$. Results for $L=W=200$, $R=0.3$ and $\rho=0.4$ considering an initial fraction of cooperators equal to $\rho_{c}(0)=0.4$ (a) and $\rho_{c}(0)=0.8(\mathrm{~b})$.

time behavior of $\rho_{c}^{e}(t)$ for the same conditions situations analyzed in figure 6. Again, the fraction of exiting defectors is higher than the expected one for small values of $P$ but the situation is reversed as $P$ increases.

As cooperators can only advantage defectors when interacting only among them, any evidence of cooperators performing better than defectors at leaving the room must be reflected in the formation of clusters of cooperators where mutual cooperation occurs. If cooperators are not clustered, the defectors will outstrip them. The effect of clustering of cooperators can be visually appreciated, but the assertion could be subjective. So we define a quantity that let us measure the instantaneous degree of clustering of cooperators by counting the fraction cooperating neighbors of each cooperating individual $i$ in the room $\omega_{c}(i, t)$ and then we define

$$
C_{c}(t)=\frac{1}{N_{c}(t)} \sum \omega_{c}(i, t) / \rho_{c}(t)
$$

where $N_{c}(t)$ and $\rho_{c}(t)$ are the number and the fraction of cooperators in the room at time $t$. We exclude isolated individuals from this count.

The results in figure 8 confirm the occurrence of the expected behavior. When the quantities $\rho_{c}^{i}(t)$ and $\rho_{c}^{e}(t)$ indicate that cooperators are performing better than defectors in reaching the exit, the results for $C_{c}(t)$ indicate an increase of the clustering of cooperators. The dynamics of the system leads to a partial segregation into cluster of cooperators and defectors, that leave the former in a situation of taking advantage from the benefits of mutual cooperation. Figure 8 shows also an interesting behavior for low values of $P$. The curves show an increase of the clustering of cooperator, more apparent for $P=1$ in Fig 1.a. This effect is different in nature and shape from those observed for higher values of $P$. The origin of this increment correspond to the fact that defectors, freely to move due to low values of punishment manage to approach the exit, displacing the cooperators and producing a segregation that isolates the last from the door. This effect is verified in Figs. 6 and 7 where we can observe that cooperator have difficulties in reaching the exit for values of $P$ close to 1 . This effect is also reflected in the fact that when the fraction of defectors is higher (0.6 in Fig. 8 a vs. 0.2 in Fig 8 b), the displacement of cooperators from the door and their corresponding segregation is enhanced. 


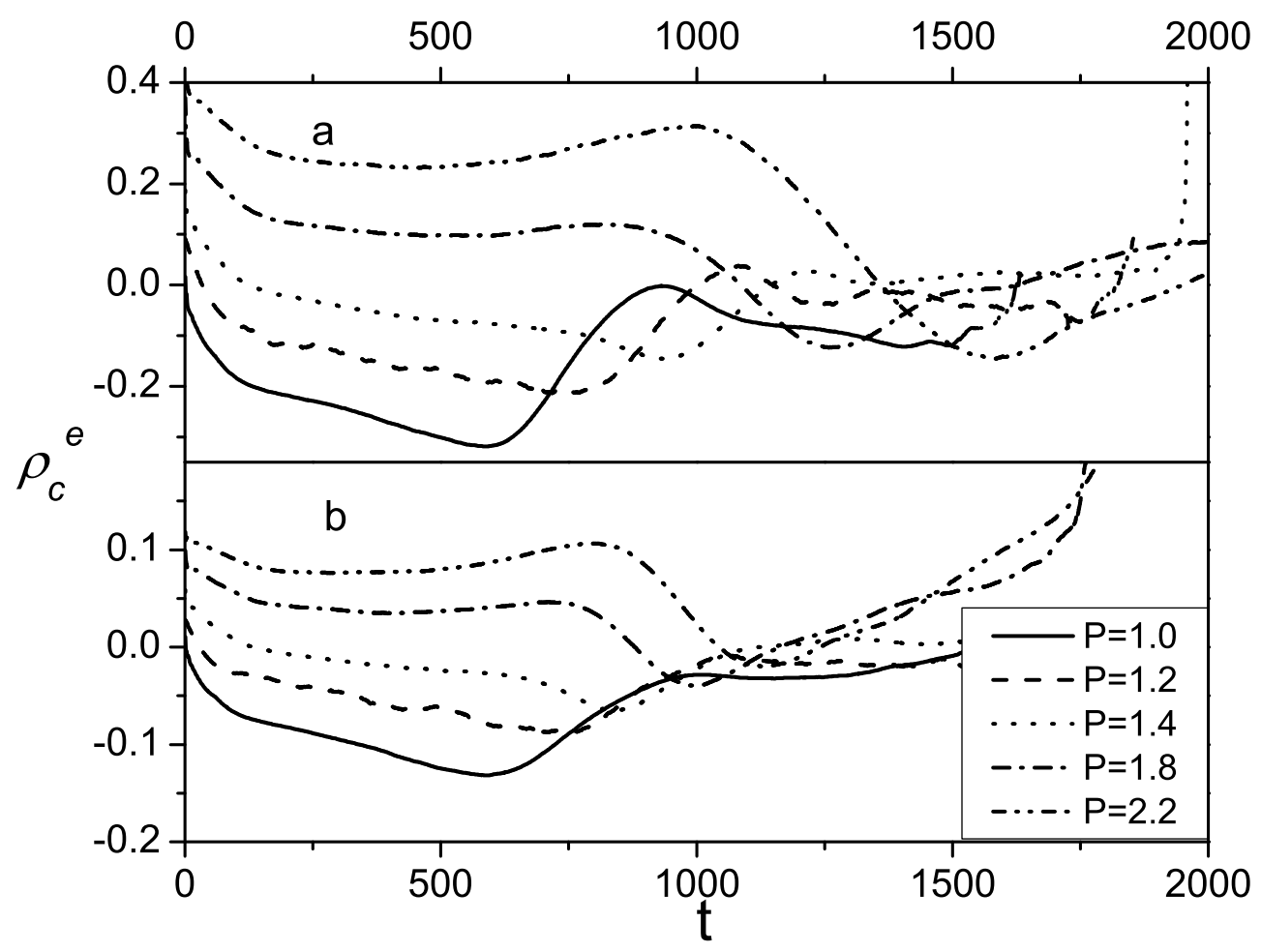

FIG. 7: Normalized fraction of cooperators exiting the room for different values of $P$. Results for $L=W=200, R=0.3$ and $\rho=0.4$ considering an initial fraction of cooperators equal to $\rho_{c}(0)=0.4(\mathrm{a})$ and $\rho_{c}(0)=0.8(\mathrm{~b})$.

The results thus indicate that cooperators can take advantage from mutual cooperation for large enough values of $P$, typically $P>1.5$, well within the PD regime where still the DC payoff is larger than the CC one. The benefits from mutual cooperation are further enhanced in the $\mathrm{SH}$ regime $(P>2)$.

\section{CONCLUSIONS}

One of the shortcomings of the Lattice Gas models used in pedestrian dynamics is the lack of inclusion of behavioral aspects. Considering this situation, the present work has a clear goal: To develop a simple model able to consider some individual attitudes that may affect the movement of interacting pedestrians. Motivated by this objective, we have amalgamated models for pedestrian evacuation with Game Theory concepts and analyzed the emergence of non trivial effects that may manifest. The results show that the emerging phenomena observed in an Evolutionary Prisoner's Dilemma are also present here. In these works 32 41] the systems are spatially extended and allow for the cooperators to form clusters capable to resist the invasion of defectors and even to expand. In the present work the evolutionary aspects has not been so far included but the growth of clusters is promoted by the mobility of the individuals. As a result of this clustering, the cooperators can profit from the only advantage they have over the defectors: the mutual cooperation. When this happens, cooperators have more success in reaching the exit than defectors, as reflected in the plots. It is important to understand the limitation and scope of the present model. We are not intending to reproduce a real situation but to point out the effects that behavioral aspects may have on the denouement of an emergency evacuation scenario. Future work envisions the inclusion of evolutionary strategies, differentiated social roles, off lattice dynamics and different geometries and obstacles. 


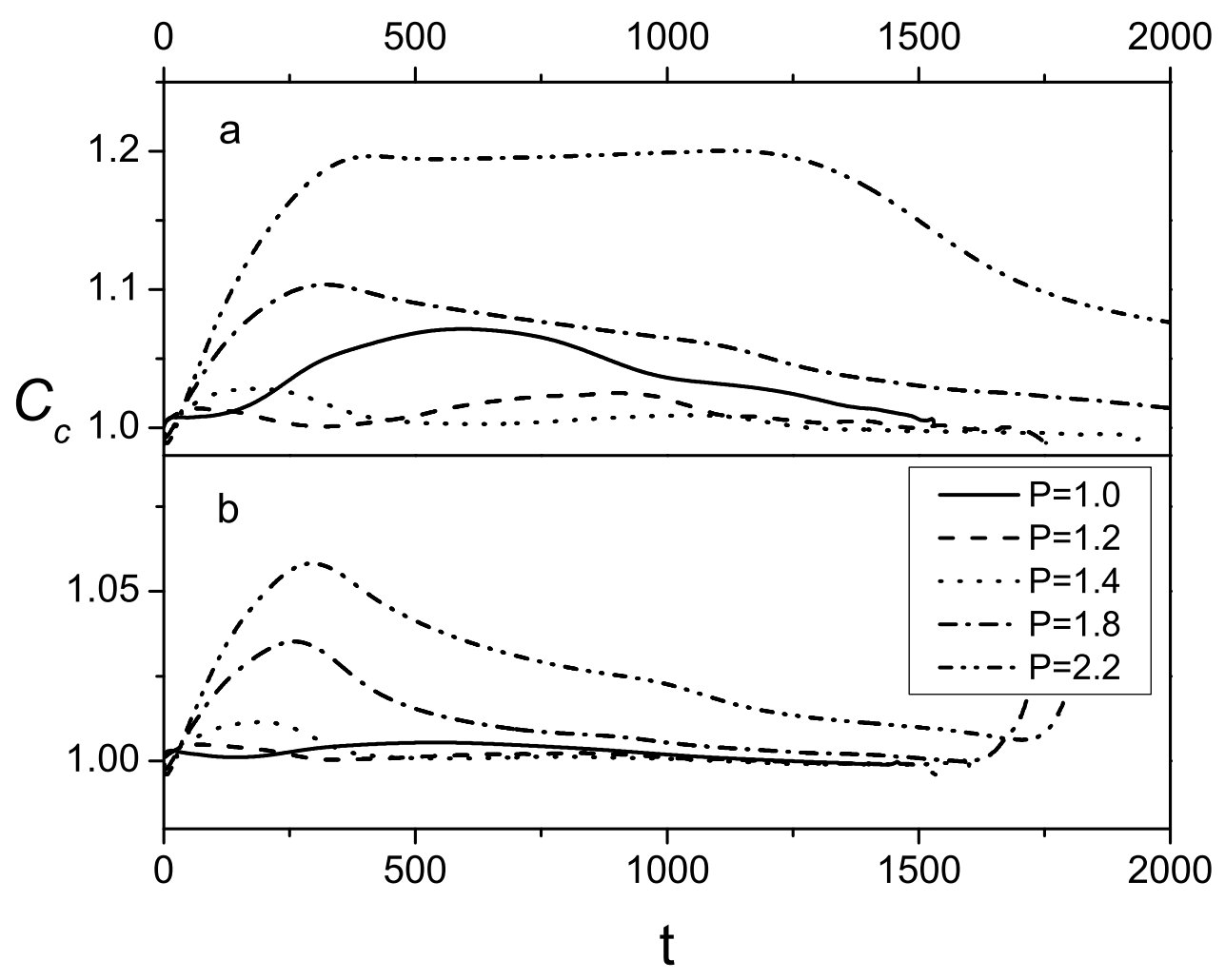

FIG. 8: Clustering of cooperators in the room for different values of $P$. Results for $L=W=200, R=0.3$ and $\rho=0.4$ considering an initial fraction of cooperators equal to $\rho_{c}(0)=0.4$ (a) and $\rho_{c}(0)=0.8(\mathrm{~b})$.

[1] D. Helbing, I. J. Farkas, P. Molnár, T Vicsek Pedestrian and Evacuation Dynamics, Ed.: M. Schreckenberg, and S. D. Sharma (Springer, Berlin) pp.21-58 (2002)

[2] D. Helbing, L. Buzna, A. Johansson, T. Werner. Transportation Science 39, 1 (2005).

[3] C. Burstedde, K. Klauck, A. Schadschneider, J. Zittartz, Physica A 295, 507 (2001).

[4] Y. Tajima and T. Nagatani, Physica A 292, 545 (2001).

[5] Kirchner and A. Schadschneider, Physica A 312, 260 (2002).

[6] D. Helbing, I. Farkas, and T. Vicsek, Nature (London) 407, 487 (2000).

[7] D. Helbing, M. Isobe, T. Nagatani, and K. Takimoto, Phys. Rev. E 67, 067101 (2003).

[8] M. Muramatsu, T. Irie, and T. Nagatani, Physica A 267, 487 (1999).

[9] V. J. Blue and J. L. Adler, J. Transp. Res. Board 1678, 135 (2000).

[10] K. Takimoto and T. Nagatani, Physica A 320, 611 (2003).

[11] D. Helbing and P. Molnár, Phys. Rev. E 51, 4282 (1995).

[12] A. Seyfried, T. Rupprecht, O. Passon, B. Steffen, W. Klingsch, and M. Boltes, Transport. Sci. 43, 395 (2009).

[13] S. Hoogendoorn and P. H. L. Bovy, Optim. Control Appl. Methods 24, 153 (2003).

[14] S. K. Baek, P. Minnhagen, S. Bernhardsson, K. Choi, and B. J. Kim, Phys. Rev. E 80, 016111 (2009).

[15] J. Tanimoto, A. Hagishima, and Y. Tanaka, Physica A 389, 5611 (2010).

[16] X. P. Zheng and Y. Cheng, Physica A 390, 1042 (2011).

[17] K. Yamamoto, S. Kokubo, and K. Nishinari, Physica A 379, 654 (2007).

[18] H. J. Huang and R. Y. Guo, Phys. Rev. E 78, 021131 (2008).

[19] A. F. Miguel, Phys. Lett. A 373, 1734 (2009).

[20] M. Chraibi, A. Seyfried, and A. Schadschneider, Phys. Rev. E 82, 046111 (2010).

[21] G. Baglietto and D. R. Parisi, Phys. Rev. E 83, 056117 (2011).

[22] G. A. Frank and C. O. Dorso, Physica A 390, 2135 (2011).

[23] L. F. Henderson, Nature (London) 229, 381 (1971).

[24] M. Fukui and Y. Ishibashi, J. Phys. Soc. Jpn. 68, 2861 (1999).

[25] U. Frisch, B. Hasslacher, Y. Pomeau, Phys. Rev. Lett. 56, 1505 (1986). 
[26] M. Muramatsu and T. Nagatani, Physica A 286, 377 (2000).

[27] A. Seyfried, B. Steffen, and T. Lippert, Physica A 368, 232 (2006).

[28] D. Helbing, R. Jiang, and M. Treiber, Phys. Rev. E 72, 046130 (2005).

[29] Q. Y. Hao, M. B. Hu, X. Q. Cheng, W. G. Song, R. Jiang, and Q. S. Wu, Phys. Rev. E 82, 026113 (2010).

[30] Q. Y. Hao, R. Jiang, M. B. Hu, B. Jia, and Q. S. Wu, Phys. Rev. E 84, 036107 (2011).

[31] A. Rapoport and M. Guyer, General Systems 11, 203 (1966).

[32] M. Doebeli and C. Hauert, Ecol. Lett. 8, 748 (2005).

[33] V. M. Eguiluz, M. G. Zimmermann, C. J. Cela-Conde, M. San Miguel, Am. J. Sociol. 110, 977 (2005).

[34] F. Fu, X. Chen, L. Liu and L Wang, Phys Lett A 371, 58 (2007).

[35] J. Gómez-Gardeñes, M. Campillo, L. M. Floría, Y. Moreno, Phys. Rev. Lett. 98, 108103 (2007).

[36] P. Langer, M.A. Nowak, C. Hauert, J. Theor. Biol 250, 634( 2008).

[37] C. Lei , J. Jia, X. Chen, R. Cong and L. Wang, Chin. Phys. Lett. 26, 080202, (2009).

[38] Szabó, G. and G. Fáth. Phys. Rep. 446, 97-216 (2007).

[39] M. N. Kuperman, S. Risau-Gusman, Phys. Rev. E 86, 016104 (2012)

[40] G. Abramson, M. Kuperman, Phys. Rev. E 63, 030901R (2001).

[41] C. P. Roca, J. A. Cuesta, A. Sánchez, Phys. Rev. E 80, 046106 (2009). 